\title{
The VIMOS VLT Deep Survey ${ }^{\star}$ the build-up of the colour-density relation
}

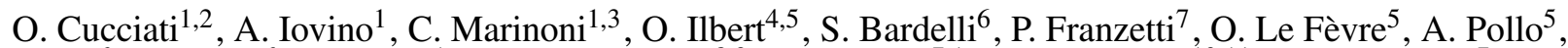 \\ G. Zamorani ${ }^{6}$, A. Cappi ${ }^{6}$, L. Guzzo ${ }^{1}$, H. J. McCracken ${ }^{8,9}$, B. Meneux ${ }^{7,1}$, R. Scaramella ${ }^{10,11}$, M. Scodeggio ${ }^{7}$, L. Tresse ${ }^{5}$, \\ E. Zucca ${ }^{6}$, D. Bottini ${ }^{7}$, B. Garilli ${ }^{7}$, V. Le Brun ${ }^{5}$, D. Maccagni ${ }^{7}$, J. P. Picat ${ }^{12}$, G. Vettolani ${ }^{10}$, A. Zanichelli ${ }^{10}$, C. Adami ${ }^{5}$, \\ M. Arnaboldi ${ }^{13}$, S. Arnouts ${ }^{5}$, M. Bolzonella ${ }^{6}$, S. Charlot ${ }^{14,8}$, P. Ciliegi $^{6}$, T. Contini ${ }^{12}$, S. Foucaud ${ }^{7}$, I. Gavignaud ${ }^{12,15}$, \\ B. Marano ${ }^{4}$, A. Mazure ${ }^{5}$, R. Merighi ${ }^{6}$, S. Paltani ${ }^{16,17}$, R. Pellò ${ }^{12}$, L. Pozzetti ${ }^{6}$, M. Radovich ${ }^{13}$, M. Bondi ${ }^{10}$, \\ A. Bongiorno ${ }^{4}$, G. Busarello ${ }^{13}$, S. de la Torre ${ }^{5}$, L. Gregorini ${ }^{10}$, F. Lamareille ${ }^{12}$, G. Mathez ${ }^{12}$, Y. Mellier ${ }^{8,9}$, \\ P. Merluzzi ${ }^{13}$, V. Ripepi ${ }^{13}$, D. Rizzo ${ }^{12}$, S. Temporin ${ }^{1}$, and D. Vergani ${ }^{7}$ \\ (Affiliations can be found after the references)
}

Received 8 March 2006 / Accepted 19 July 2006

\begin{abstract}
We investigate the redshift and luminosity evolution of the galaxy colour-density relation using the data from the First Epoch VIMOS-VLT Deep Survey (VVDS). The size (6582 galaxies with good quality redshifts), depth $\left(I_{A B} \leq 24\right)$ and redshift sampling rate (20\% on the mean) of the survey enable us to reconstruct the 3D galaxy environment on relatively local scales $\left(R=5 h^{-1} \mathrm{Mpc}\right)$ up to redshift $z \sim 1.5$. Particular attention has been devoted to calibrate a density reconstruction scheme, which factors out survey selection effects and reproduces in an unbiased way the underlying "real" galaxy environment. We find that the colour-density relation shows a dramatic change as a function of cosmic time. While at lower redshift we confirm the existence of a steep colour-density relation, with the fraction of the reddest(/bluest) galaxies of the same luminosity increasing(/decreasing) as a function of density, this trend progressively disappears in the highest redshift bins investigated. Our results suggest the existence of an epoch (more remote for brighter galaxies) characterized by the absence of the colour-density relation on the $R=5 h^{-1} \mathrm{Mpc}$ scales investigated. The rest frame $u^{*}-g^{\prime}$ colour-magnitude diagram shows a bimodal pattern in both low and high density environments up to redshift $z \sim 1.5$. We find that the bimodal distribution is not universal but strongly depends upon environment: at lower redshifts the colour-magnitude diagrams in low and high density regions are significantly different while the progressive weakening of the colour-density relation causes the two bimodal distributions to nearly mirror each other in the highest redshift bin investigated. Both the colour-density and the colour-magnitude-density relations, on the $R=5 h^{-1} \mathrm{Mpc}$ scales, appear to be a transient, cumulative product of genetic and environmental factors that have been operating over at least a period of 9 Gyr. These findings support an evolutionary scenario in which star formation/gas depletion processes are accelerated in more luminous objects and in high density environments: star formation activity is progressively shifting with cosmic time towards lower luminosity galaxies (downsizing), and out of high density environments.
\end{abstract}

Key words. cosmology: observations - large scale structure of Universe - galaxies: distances and redshifts - galaxies: evolution galaxies: statistics - galaxies: fundamental parameters

\section{Introduction}

There is a well known connection between galaxy properties such as morphology, luminosity, integral colour, specific star formation rate (SFR), surface brightness and the local environment wherein galaxies reside (e.g., Spitzer \& Baade 1951; Dressler 1980; Whitmore et al. 1993; Poggianti et al. 1999; Marinoni et al. 1999, 2002; Balogh et al. 2004a,b; Hogg et al. 2004; Blanton et al. 2005; Weinmann et al. 2006). These correlations extend smoothly over a wide range of density enhancements,

\footnotetext{
* Based on observations obtained with the European Southern Observatory Very Large Telescope, Paranal, Chile. This paper also uses observations obtained with MegaPrime/MegaCam, a joint project of CFHT and CEA/DAPNIA, at the Canada-France-Hawaii Telescope (CFHT) which is operated by the National Research Council (NRC) of Canada, the Institut National des Science de l'Univers of the Centre National de la Recherche Scientifique (CNRS) of France, and the University of Hawaii. This work is based in part on data products produced at TERAPIX and the Canadian Astronomy Data Centre as part of the Canada-France-Hawaii Telescope Legacy Survey, a collaborative project of NRC and CNRS.
}

from the extreme environment of rich clusters to very low densities, well beyond the region where the cluster environment is expected to have much influence (e.g., Postman \& Geller 1984; Zabludoff \& Mulchaey 1998; Gómez et al. 2003).

Despite increasing precision in quantifying environmental correlations, we still lack a satisfactory understanding of where these trends stem from. Several physical mechanisms are expected to be crucial in determining the properties of galaxies in over-dense regions: ram pressure stripping of gas (Gunn \& Gott 1972), galaxy-galaxy merging (Toomre \& Toomre 1972), strangulation (Larson et al. 1980) and harassment (Moore et al. 1996). Although these processes are plausible, each mechanism has specific environments and timescales in which it operates most effectively, and additional ingredients may turn out to be essential for progressing towards a coherent physical interpretation of the observations. For example, environmental analyses have yet to elucidate the relative and complementary importance of the physics regulating galaxy formation. It is not yet clear to what extent local phenomena such as feedbacks from supernovae and central black holes do contribute to the observed 
density dependence of the galaxy structural parameters. Moreover, it is known that in a Gaussian random field there is a statistical correlation between mass fluctuations on different scales, with most massive halos preferentially residing within over-densities on larger scales (see Kaiser 1987; Mo \& White 1996). What is less clear is the role of initial cosmological conditions in triggering the observed density dependence of optical galaxy properties (Abbas \& Sheth 2005).

A key question that still needs to be addressed is whether these environmental dependencies were established early on when galaxies first assembled, or whether they are the present day cumulative end product of multiple processes operating over a Hubble time. In other words if these dependencies arise during the formation of galaxies (the so-called "nature" hypothesis) or whether they are caused by density-driven evolution (the "nurture" scenario).

A promising approach to addressing these issues involves extending observations beyond the local universe. The relations between environment and galaxy properties have still virtually no empirical constraints beyond $z \sim 0.5$, except in cluster of galaxies (e.g., Smith et al. 2005; Postman et al. 2005; Tanaka et al. 2005). A preliminary analysis over volumes which average over many different environments has been attempted out to $z=1$ by Nuijten et al. (2005), using a photometric sample of galaxies. However it has been recently stressed that environmental investigations crucially require high resolution spectral measurements of galaxy positions. For example, Cooper et al. (2005) find that even optimistic photometric redshift errors $(\sigma=0.02)$ smear out the galaxy distribution irretrievably on small scales, significantly limiting the application of photometric redshift surveys to environment studies (but see for a particular case Guzzo et al. 2006).

Large and deep redshift surveys of the universe are the best available instrument to select a representative sample of the galaxy population over a broad and continuous range of densities and cosmic epochs. Moreover these surveys open up the possibility of exploring such trends in different magnitude bands. In this study, we use the VIMOS VLT Deep Survey (Le Fèvre et al. 2005), the largest (6582 objects with secure redshifts), deepest $(0.05<z<5.0)$, purely-magnitude selected $\left(I_{A B} \leq 24\right)$ redshift sample currently available, to explore the colour-density relation as a function of both luminosity and cosmic time. In particular the main goal of this investigation is to portray the colour-density relation at different epochs and evaluate eventual changes in its overall normalization (Butcher \& Oemler effect, Butcher \& Oemler 1984) and slope (Dressler effect, Spitzer \& Baade 1951; Dressler 1980).

While redshift surveys have grown in scale and environmental studies have acquired momentum, much less attention has been devoted to investigate how the various systematics introduced by the particular survey observing strategies may affect the estimation of environment. In our study, we pay special attention to constrain the parameter space where the VVDS density field reproduces in a statistically unbiased way the underlying parent density field.

This paper is set out as follows: in Sect. 2 we briefly describe the first-epoch VVDS-0226-04 data sample. In Sect. 3 we introduce the technique applied for reconstructing the threedimensional density field traced by VVDS galaxies, providing details about corrections for various selection effects. In Sect. 4 we test the statistical representativity of the reconstructed VVDS density field using mock catalogues. We present our results on the dependence of galaxy colours from local density in Sect. 5 and discuss them in Sect. 6. Conclusions are drawn in Sect. 7.
The coherent cosmological picture emerging from independent observations and analyses motivates us to frame all the results presented in this paper in the context of a flat, vacuum dominated cosmology with $\Omega_{\mathrm{m}}=0.3$ and $\Omega_{\Lambda}=0.7$. Throughout, the Hubble constant is parameterized via $h=H_{0} / 100$. All magnitudes in this paper are in the AB system (Oke \& Gunn 1983), and from now on we will drop the suffix $A B$.

\section{The first-epoch VVDS redshift sample}

The primary observational goal of the VIMOS-VLT Deep Survey as well as the survey strategy and first-epoch observations in the VVDS-0226-04 field (from now on simply VVDS-02h) are presented in Le Fèvre et al. (2005), hereafter Paper I.

Here it is enough to stress that, in order to minimize selection biases, the VVDS survey in the VVDS-02h field has been conceived as a purely flux-limited $(17.5 \leq I \leq 24)$ survey, i.e. no target pre-selection according to colours or compactness was implemented. Stars and QSOs have been a posteriori removed from the final redshift sample. Photometric data in this field are complete and free from surface brightness selection effects, up to the limiting magnitude $I=24$ (McCracken et al. 2003; Le Fèvre et al. 2004a). $B, V, R, I$ photometry was acquired with the widefield $12 \mathrm{~K}$ mosaic camera at the CFHT, while $u^{*}, g^{\prime}, r^{\prime}, i^{\prime}, z^{\prime}$ photometry is part of the Canada-France-Hawaii Telescope Legacy Survey.

First-epoch spectroscopic observations in the VVDS-02h field were carried out using the VIMOS multi-object spectrograph (Le Fèvre et al. 2003) during two runs between October and December 2002 (see Paper I). VIMOS observations have been performed using 1 arcsec wide slits and the LRRed grism, which covers the spectral range $5500<\lambda(\AA)<9400$ with an effective spectral resolution $R \sim 227$ at $\lambda=7500 \AA$. The accuracy in redshift measurements is $\sim 275 \mathrm{~km} \mathrm{~s}^{-1}$. Details on observations and data reduction are given in Paper I, and in Le Fèvre et al. (2004b).

The first-epoch VVDS-02h data sample extends over a sky area of $0.7 \times 0.7 \mathrm{deg}^{2}$, which was targeted according to a 1 , 2 or 4 passes strategy, i.e. giving to any single galaxy in the field 1, 2 or 4 chances to be targeted by VIMOS (see Fig. 12 of Paper I), and has a median redshift of about $z \sim 0.76$. It contains 6582 galaxies with secure redshifts, i.e. redshift determined with a quality flag $\geq 2$, see Paper I (5882 with $0.25 \leq z \leq 1.5$ ) and probes a comoving volume (up to $z=1.5$ ) of nearly $1.5 \times 10^{6} h^{-3} \mathrm{Mpc}^{3}$ in a standard $\Lambda \mathrm{CDM}$ cosmology. This volume has transversal dimensions $\sim 37 \times 37 h^{-1} \mathrm{Mpc}$ at $z=1.5$ and extends over $3060 \mathrm{~h}^{-1} \mathrm{Mpc}$ in radial direction.

For this study we define also a sub-sample (VVDS-02h-4P) with galaxies selected in a contiguous sky region of $0.4 \times$ $0.4 \mathrm{deg}^{2}$ which has been homogeneously targeted four times by VIMOS observations. The VVDS-02h-4P subsample contains 2903 galaxies with secure redshift (2647 with $0.25 \leq z \leq 1.5$ ) and probes one-third of the total VVDS-02h volume.

\section{Environment reconstruction scheme}

To study environmental effects on galaxy properties, we need to define an appropriate density estimator which properly corrects for all the survey selection biases. 
We characterize the environment surrounding a given galaxy at comoving position $\boldsymbol{r}$, by means of the dimensionless $3 \mathrm{D}$ density contrast smoothed over a typical dimension $R$ :

$\delta(\boldsymbol{r}, R)=\frac{\rho(\boldsymbol{r}, R)-\bar{\rho}(\boldsymbol{r})}{\bar{\rho}(\boldsymbol{r})}$,

where $\rho(\boldsymbol{r}, R)$ is the number density of galaxies brighter than a fixed absolute magnitude threshold $\mathcal{M}^{c}$ and $\bar{\rho}(\boldsymbol{r})$ is the mean density at position $\boldsymbol{r}$. The smoothed number density of galaxies on a scale $R$ is estimated as an appropriately weighted convolution between Dirac's delta functions and some arbitrary filter $F$ :

$\rho\left(\boldsymbol{r}, R,<\mathcal{M}^{c}\right)=\sum_{i} \frac{\delta^{D}\left(\boldsymbol{r}-\boldsymbol{r}_{i}\right) * F\left(\frac{\left|\boldsymbol{r}-\boldsymbol{r}_{i}\right|}{R}\right)}{S\left(r_{i}, \mathcal{M}^{c}\right) \Phi(m) \zeta(z, m) \Psi(\alpha, \delta)}$,

where the sum is taken over all the galaxies excluding that at position $\boldsymbol{r}$ (the one for which we estimate the surrounding environment) and where $F(|\boldsymbol{r}| / R)$ is the window-function, which in this study is modeled in terms of a normalized Gaussian filter:

$F\left(\frac{|\Delta \boldsymbol{r}|}{R}\right)=\frac{1}{\left(2 \pi R^{2}\right)^{3 / 2}} \exp \left[-\frac{1}{2}\left(\frac{|\Delta \boldsymbol{r}|}{R}\right)^{2}\right]$.

Note that, for example, with the choice $R=5 h^{-1} \mathrm{Mpc}$ we smooth the galaxy distribution over an effective volume which roughly corresponds to that enclosed in spheres of radius $8 h^{-1} \mathrm{Mpc}$.

As shown by Ilbert et al. (2005), $\bar{\rho}(\boldsymbol{r})$ evolves by nearly a factor of 2 for galaxies brighter than $\mathcal{M}_{\mathrm{B}}^{*}(z=0)$ from redshift $z=0$ to redshift $z=1$. We thus compute the characteristic mean density at position $\boldsymbol{r}$ with Eq. (2) by simply averaging the galaxy distribution in survey slices $r \pm R_{\mathrm{S}}$, with $R_{\mathrm{S}}=400 h^{-1} \mathrm{Mpc}$ (see Marinoni et al. 2005).

Finally, the four functions in the denominator of Eq. (2) correct for various survey observational characteristics:

- $S\left(r, \mathcal{M}^{c}\right)$ is the distance-dependent selection function of the sample which corrects for the sample progressive radial incompleteness. Since our spectroscopic sample is limited at bright and faint apparent magnitudes (17.5 $\leq I \leq 24)$, at any given redshift we can only observe galaxies in a specific, redshift-dependent, absolute magnitude range. The function $S\left(r, \mathcal{M}^{c}\right)$ is computed using the galaxy luminosity function derived by Ilbert et al. (2005) and assuming $\mathcal{M}^{c}=-15+5 \log h$. A thorough discussion of this function can be found in Marinoni et al. (2005).

- $\Phi(m)$ corrects for the slight bias against bright objects introduced by the slit positioning tool VMMPS/SPOC (Bottini et al. 2005).

$-\zeta(z, m)$ is the correction for the varying spectroscopic success rate as a function of the apparent $I$ magnitude and of $z$ itself (see Ilbert et al. 2005).

- $\Psi(\alpha, \delta)$ is a correction for the uneven spectroscopic sampling of the VVDS on the sky, depending on the different number of passes done by the VIMOS spectrograph in different sky regions.

The density field reconstructed using Eq. (2) has the advantage of exploiting all the galaxies in our sample. In principle, the environment is thus defined as the fluctuation field traced at every redshift by galaxies as fainter as $\mathcal{M}^{c}=-15+5 \log h$.

The assumption implicit in this reconstruction scheme is that the subset of galaxies luminous enough to enter our flux-limited sample at a given redshift are fair tracers of the full population of galaxies. With this assumption we neglect possible biases due to the dependence of clustering on luminosity; moreover, adopting a universal luminosity function we do not take into account a dependence of the LF on morphological type and environment. Systematic errors (increasing with redshift) could also be introduced as a consequence of errors in the sample selection function. Such problems are unavoidable when dealing with a flux-limited sample.

Therefore as a complementary approach we have reconstructed the density field using a volume-limited subsample of galaxies. This approach overcomes all the above limitations and gives us the possibility to test the robustness of our results against different modelling strategies. The price to be paid is obviously the much smaller number of galaxies; we also neglect possible effects due to the evolution of the LF, particularly its faint end: this means that clumps identified in the density field of luminous galaxies could have a different density of fainter galaxies as a function of redshift.

The advantage is that the two approaches suffer from different limitations, and obtaining consistent results with both of them allows us to derive more robust conclusions.

We will now discuss in some detail how we computed the three functions $\Phi(m), \zeta(z, m)$ and $\Psi(\alpha, \delta)$.

\subsection{VMMPS/SPOC sampling rate as a function of apparent magnitude}

In the VVDS, as in most redshift surveys, only a fraction of all galaxies in the photometric sample satisfying the given flux limit criteria is targeted: nearly $40 \%$ for 4 passes area, and a lower fraction for 3, 2 and 1 pass areas (see Paper I). Furthermore only a fraction of the targeted objects yields a reliable, i.e. quality flag $\geq 2$, redshift: $\sim 80 \%$ (see Paper I).

Since the VVDS targeting strategy is optimized to maximize the number of slits on the sky, the selection of faint objects is systematically favoured (Bottini et al. 2005; Pollo et al. 2005; Ilbert et al. 2005). As a consequence, the final spectroscopic sample is slightly biased with respect to the photometric one, at the bright magnitude end. We thus compute the correcting function $\Phi(\mathrm{m})$ as the ratio of the distribution of the magnitudes of targeted objects in all VVDS-02h data to the distribution of the magnitudes of photometric catalogue.

\subsection{The redshift sampling rate as a function of apparent magnitude and redshift}

The function $\zeta(z, m)$ provides a correction for two effects: the progressive degradation toward fainter magnitudes of our ability of measuring a redshift and the presence of redshift ranges where the number and strength of identifiable spectral features is scarce. To determine this function we followed the approach outlined in Ilbert et al. (2005), and we refer the reader to that paper for details.

Here is enough to mention that the weights introduced by this function are determined by comparing the photometric redshift distribution of all targeted galaxies with the spectroscopic redshift distribution of all high quality flag galaxies, i.e. quality flag $\geq 2$. This comparison is done in the subset area of the VVDS-02h field where $J$ and $K$ photometry is available (Iovino et al. 2005), as the availability of near-infrared photometry improves the robustness of photometric redshift estimates. 

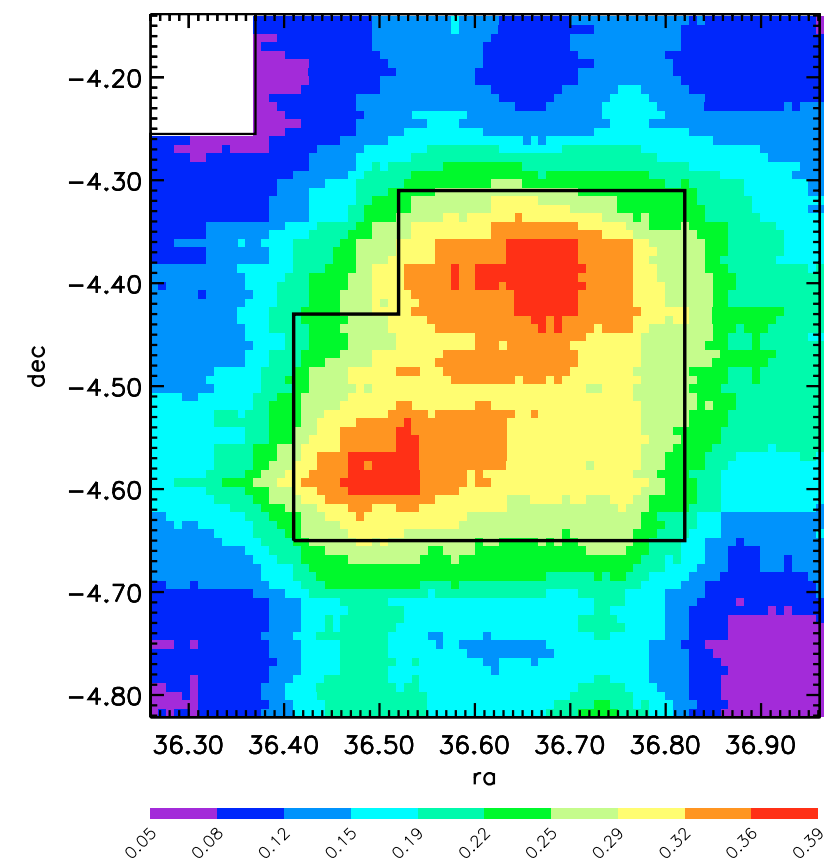

Fig. 1. The function $\Psi(\alpha, \delta)$ over the full VVDS-02h field, before normalization. The grid used had steps $s_{\text {step }}=30^{\prime \prime}$ in Right Ascension and Declination, and for each grid position we used squares of size $s_{\text {box }}=7^{\prime}$ to estimate $\Psi(\alpha, \delta)$. Both the central area covered by 4 VIMOS passes (highlighted by the central continuous line) and the missing $\mathrm{N}-\mathrm{W}$ corner are clearly visible.

\subsection{Angular sampling rate}

The function $\Psi(\alpha, \delta)$ further modulates the sampling rate defined by the two previous functions. Its purpose is to make allowance for the number of passes performed by VIMOS in the $(\alpha, \delta)$ region considered.

This function was calculated in two steps. In a grid of step size $s_{\text {step }}=30^{\prime \prime}$ in Right Ascension and Declination over the full VVDS-02h field, we computed in squares of size $s_{\text {box }}=7^{\prime}$ (which roughly corresponds to the dimensions of a VIMOS quadrant) the ratio of the number of objects with a reliable redshift to the number of potential targets in the same area. This ratio provides for each grid point the global sampling rate of the VVDS, irrespective of magnitude and redshift. $\Psi(\alpha, \delta)$ was then obtained by normalizing to unity the mean value of this ratio over the full $\alpha, \delta$ range of the survey.

Figure 1 shows in colour-scale the function $\Psi(\alpha, \delta)$ before normalization over the full VVDS-02h field. The central area covered by 4 VIMOS passes is clearly visible, and corresponds to a sampling rate of $33 \%$. In other words in the VVDS- $02 \mathrm{~h}-4 \mathrm{P}$ area, and down to our selection limit $I \leq 24.0$, on the mean one galaxy out of three gets a reliable redshift. The slightly unevenness in the coverage within the VVDS-02h-4P area is due to some lower quality quadrants. The sampling rate declines going to region covered by 3,2 and 1 VIMOS passes. Also the missing $\mathrm{N}-\mathrm{W}$ corner is clearly visible.

We explored smaller values for $s_{\text {box }}$, down to $1^{\prime}$, without any significant improvement of the results obtained (see later Sect. 4). Our final choice for $s_{\text {box }}$, being comparable with the size of a VIMOS quadrant $\left(7^{\prime} \times 8^{\prime}\right)$, enables us to take into account the presence of missing or poorer quality quadrants within a pointing. In our computation of $\Psi(\alpha, \delta)$ we suitably adjusted $s_{\text {box }}$ for grid positions near the field boundaries to avoid introducing spurious border effects.

\subsection{Boundary effects}

The first-epoch VVDS-02h data sample extends over a square area of $0.7 \times 0.7 \mathrm{deg}^{2}$ except for a small corner missing, the North-West one (see Fig. 1).

The effect of the presence of edges is to surreptitiously lower the measured density. To take into account this problem we scaled the densities measured around each galaxy dividing by the fraction of the volume of the filter contained within the survey borders. Such a correction can be quite large, especially for galaxies located near to the survey borders, for example those lying at the corners in Right Ascension and Declination of our survey layout. To minimize this correction, and considering that the border regions of the survey are those with the lowest sampling rate, we introduced a further trimming: in all our plots we considered only galaxies in positions such that at least $50 \%$ of the volume of the 3-D Gaussian used to define the density contrast lies inside the volume surveyed. These galaxies, for $R=5 h^{-1} \mathrm{Mpc}\left(R=8 h^{-1} \mathrm{Mpc}\right)$, amount to $10 \%$ (30\%) of our sample down to $\left(M_{B}-5 \log h\right) \leq 19.0$.

Scattered through the entire survey field there are further small spurious voids due to masking in the photometric catalogue of areas contaminated by bright stars and diffraction spikes (for a total masked area of less than $10 \%$ of the total area). Our simulations show that neglecting these spurious voids has no significant impact on our ability to reconstruct the underlying density contrast field.

\section{Estimating reconstruction systematics using mock catalogues}

We made extensive use of simulations in order to explore the redshift ranges and smoothing length scales $R$ over which our density reconstruction scheme (cf. Eq. (2)) is not affected by the specific VVDS observational constraints. These include intrinsic limitations in recovering real space positions of galaxies (peculiar velocities contaminations, spectroscopic accuracy...), survey geometrical constraints, sampling and instrumental selections effects.

To this purpose we used mock catalogues extracted from GalICS (Galaxies in Cosmological Simulations) which is a numerical model of hierarchical galaxy formation that combines cosmological simulations of dark matter with semi-analytic prescriptions for galaxy formation (Hatton et al. 2003). Thanks to the implementation of the Mock Map Facility (MoMaF, Blaizot et al. 2005) we converted the 3D mock catalogues into 2D sky images, and handled the $2 \mathrm{D}$ projection of the simulation as a pseudo-real imaging survey.

Using an approach similar to that described in Pollo et al. (2005) and in Marinoni et al. (2005) we constructed VVDSlike mocks which reproduce the survey angular extension, volume, flux constraints and spectroscopic resolution. We refer to these samples, which one would ideally obtain by observing with $100 \%$ sampling rate the VVDS-02h field, as the parent catalogues. These parent catalogues are unaffected by the presence of boundaries (they have an angular extension much wider than the VVDS-02h field) and they do not contain masked areas.

To each of these parent catalogues we applied the various instrumental selection effects and the VVDS observing strategy, including the same geometrical pattern of excluded regions with which we avoided to survey sky areas contaminated by the presence of bright stars or photometric defects, the same target selection procedure and the same magnitude-distribution of failures in redshift measurements (see Paper I). In this way we closely 


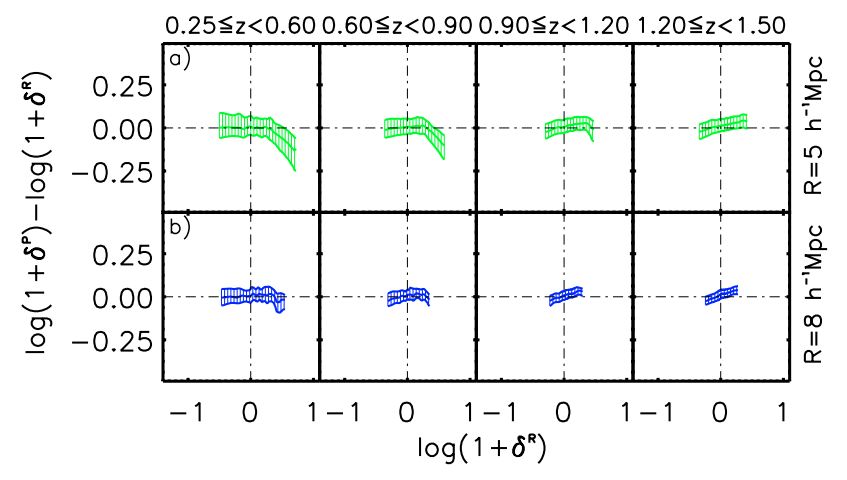

Fig. 2. The difference between over-densities reconstructed in redshift space $\left(\delta^{\mathrm{P}}\right)$ and in real space $\left(\delta^{\mathrm{R}}\right)$ is plotted as a function of the real space density contrast. Results represent the average of 50 pseudo independents GalICS mocks. Columns are for four redshift bins $(0.25<$ $z \leq 0.6,0.6 \leq z \leq 0.9,0.9 \leq z \leq 1.2$ and $1.2 \leq z \leq 1.5)$ and rows for two different values of the smoothing scale: $R=5(/ 8) h^{-1}$ Mpc from top to bottom. Central solid lines represent continuous median values computed in equipopulated bins, and the boundaries of shaded areas show the 25 th and the 75 th percentiles.

matched the actual characteristics of the VVDS-02h field as observed, and the resulting catalogues are called observed catalogues.

Finally, we also constructed catalogues in real space, that is catalogues with the same objects as the parent catalogues, but without implementing the effects of large scale streaming motions and measurement errors in the redshift estimate.

The density contrast was therefore reconstructed according to the prescriptions described in Sect. 3 for parent $\left(\delta^{\mathrm{P}}(\boldsymbol{r}, R)\right)$, real space $\left(\delta^{\mathrm{R}}(\boldsymbol{r}, R)\right)$ and observed catalogues $\left(\delta^{\mathrm{O}}(\boldsymbol{r}, R)\right)$.

Our comparison strategy is twofold. First we check to what extent non-cosmological kinematical effects such as galaxy peculiar velocities and spectroscopic random errors in redshift measurements smear out the galaxy cosmological redshift and hamper our ability to recover real space galaxy positions and small scale environmental densities. Second we test how geometrical artifacts introduced by the specific VVDS target selection strategy and its sparseness (only 1 galaxy over three at the VVDS magnitude depth has a measured redshift in the four passes area) degrade the underlying signal.

There are a few obvious guidelines in selecting the ranges of plausible parameters for reliable density reconstruction. First of all, one may expect to exclude smoothing lengths $R \ll$ $4 h^{-1} \mathrm{Mpc}$, i.e. much smaller than the mean inter-particle separation of our sample, which is $4.4 h^{-1} \mathrm{Mpc}$ at the peak of the observed redshift distribution. We tested, for example, that on these scales a substantial fraction of galaxies that are classified as isolated (i.e. with no observed neighbour in the reference volume considered) in the observed catalogue is not constituted by truly isolated galaxies in the parent catalogue, but by galaxies populating over-dense regions $(\delta \geq 0)$ for which the density field reconstruction fails. We also excluded from the analysis regions with redshift greater than $z=1.5$, where the sample becomes too sparse and the mean inter-galaxy separation too large. Similarly, the lower redshift limit $z_{t}$ is set by imposing that the transversal dimension $L$ of the field be $L\left(z_{t}\right)>R$. As an example, for a Gaussian window of size $R=8 h^{-1} \mathrm{Mpc}$, we have $z_{t} \sim 0.25$.

Figure 2 shows the difference between densities reconstructed in the parent catalogue $\left(\delta^{\mathrm{P}}\right)$ and in real space $\left(\delta^{\mathrm{R}}\right)$ versus the real space density contrast. We see that on scales $R=5 h^{-1} \mathrm{Mpc}$, the parent catalogue (that is redshift-space)

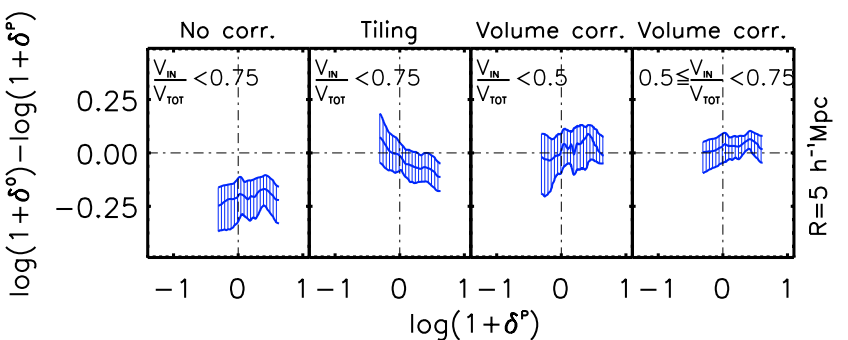

Fig. 3. The difference between over-densities reconstructed in the observed $\left(\delta^{\mathrm{O}}\right)$ and parent $\left(\delta^{\mathrm{P}}\right)$ catalogue is plotted as a function of the parent density contrast $\delta^{\mathrm{P}}(\boldsymbol{r}, R)$. The four panels correspond to different strategies of corrections for border effects, as indicated by the label on the top. $V_{\text {in }} / V_{\text {tot }}$ in each panel shows the limit on the fraction of the volume of the Gaussian filter $\mathrm{F}$ contained within the survey borders for each of the galaxies plotted. Central solid lines represent continuous median values computed in equipopulated bins, and the boundaries of shaded areas show the 25 th and the 75 th percentiles. See text for more details.

densities are systematically underestimated in over-dense regions. This is because, on small scales, non-linear structures observed in redshift space are smeared out along the line of sight (the so-called Finger of God effect). On larger scales we expect the opposite (Kaiser effect), i.e. that the infalling pattern towards over-dense regions spuriously enhances the density contrast recovered in redshift space. Effectively, the transition between these two different regimes becomes appreciable on scales $R=8 h^{-1} \mathrm{Mpc}$.

For our study as a function of environment, it is of fundamental importance to differentiate in a robust way between over- and under-dense regions, and Fig. 2 shows that underdense regions are safely recovered in redshift space. Moreover, the small amount of the underestimation in over-dense regions (for $R \geq 5 h^{-1} \mathrm{Mpc}$ ) guarantees that there is no fictitious percolation of high density into low density environments and viceversa.

A possible concern in our density reconstruction scheme could be on systematics introduced by our chosen strategy to correct for survey borders. Our survey is not a pencil beam survey in the original sense, but still its transverse dimensions are much smaller than its dimension along the redshift axis. Figure 3 shows the difference between observed and parent density contrast $\delta$ as a function of the parent $\delta^{\mathrm{P}}$ for three possible strategies of border effects corrections in an observed catalogue that is like the parent except for the presence of VVDS-like borders. In this plot we show only galaxies such that the fraction $V_{\mathrm{IN}} / V_{\mathrm{TOT}}$ of the volume of the Gaussian filter $F$ contained within the survey borders is less than a fixed value, as indicated in each panel.

In the first panel no correction for borders is implemented and, as expected, a systematic underestimate of the parent density is clearly visible for border galaxies. In the second panel we show the results obtained when applying a tiling correction. We tiled around the observed VVDS-02h field 8 replicas of the VVDS-02h field itself (after adding a smaller tile to cover the missing N-W corner, see Fig. 1). We tested the robustness of the tiling correction as follows: we tiled the mocks simulating the VVDS-02h field and compared the recovered densities with the underling "true" densities of the parent simulations which have a larger extension than the VVDS field. The tiling strategy allows a more reliable reconstruction of the environment, at least in a statistical way, for galaxies near the edges of the survey. Anyway, still a non negligible underestimate of overdense regions and overestimate of underdense regions is visible. This 


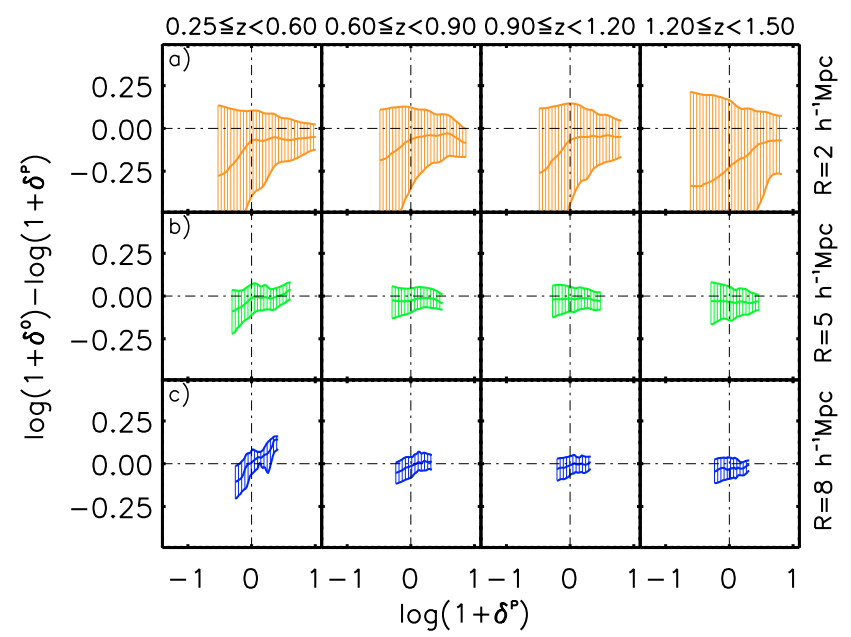

Fig. 4. The difference between over-densities reconstructed in the observed and parent catalogue is plotted as a function of the parent density contrast $\delta^{\mathrm{P}}(\boldsymbol{r}, R)$. Columns are for four redshift bins $(0.25<z \leq$ $0.6,0.6 \leq z \leq 0.9,0.9 \leq z \leq 1.2$ and $1.2 \leq z \leq 1.5)$ and rows for three different values of $R: 2,5,8 h^{-1} \mathrm{Mpc}$ from top to bottom. Central solid lines represent continuous median values computed in equipopulated bins, and the boundaries of shaded areas show the 25th and the 75th percentiles. Results represent the average of 50 pseudo-independent GalICS mocks.

is due to the fact that the tiling smeares the original densitities by adding for the border objects a random density to the actual density in the parent catalogue. Finally the last two panels show the results of a volume correction scheme. We correct the density contrast measured around each observed galaxy by a factor that corresponds to the inverse of the fraction of the volume of the Gaussian filter $F$, centered on the galaxy in question, inside the VVDS-like mock survey. By comparing the reconstructed density with the "real" density field of the parent simulation we conclude that there is no large, systematic shift between observed and parent $\delta$, and especially so when excluding all galaxies such that this correction is higher than 2 as in the last panel. This is the solution we adopted.

Finally we turn to the question of assessing how well the observed galactic environment, reconstructed after applying the whole VVDS pipeline to simulations, traces the underlying parent over-density field. In Fig. 4 we plot the difference between the logarithm of the observed and of parent over-densities $(\Delta=$ $\left.\log \left(1+\delta^{\mathrm{O}}(\boldsymbol{r}, R)\right)-\log \left(1+\delta^{\mathrm{P}}(\boldsymbol{r}, R)\right)\right)$ with respect to the logarithm of the density contrast $\log \left(1+\delta^{\mathrm{P}}(\boldsymbol{r}, R)\right)$ as a function of redshift. Rows refer to three different values of $R: 2,5,8 h^{-1} \mathrm{Mpc}$ from top to bottom. In this plot and in all subsequent plots we followed the recipe discussed in Sect. 3.4 for border corrections and used only galaxies located in a position with respect to survey borders such that less than $50 \%$ of the volume of the 3-D Gaussian centered on them and used to define the density contrast lies outside the volume surveyed.

In this way we can check for possible systematics affecting the observed over-density: e.g., a systematic over/underestimate of density contrast introduced by the wrong functions in Eq. (2), or by non-trivial border effects introduced by masking of some areas of the observed catalogue. It is evident how badly we reconstruct the density field on scales as small as $2 h^{-1} \mathrm{Mpc}$, as expected from the previous discussion. For $R \geq 5 h^{-1} \mathrm{Mpc}$, there is no evidence of systematic biases in the reconstruction of the over-density distribution. This conclusion holds irrespective of the particular redshift range investigated in the interval $0.25<z<1.5$.

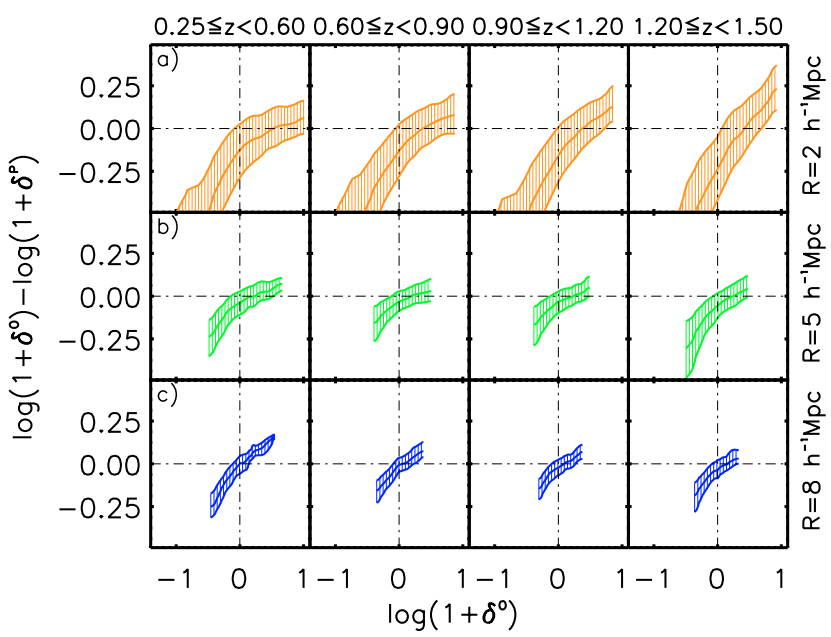

Fig. 5. As in Fig. 4, but here the difference between over-densities reconstructed in the observed and parent catalogue is plotted as a function of the observed variable $\delta^{\mathrm{O}}(\boldsymbol{r}, R)$.

However, using real data, we have only access to the information contained in the observed over-density. The conditional distribution of $\delta$ given the observed over-density $\delta^{\mathrm{O}}\left(P\left(\Delta \mid \delta^{\mathrm{O}}\right)\right)$ is expected to be different from $P\left(\Delta \mid \delta^{\mathrm{P}}\right)$. Therefore, we analyzed the difference between observed and parent over-densities as a function of $\delta^{\mathrm{O}}(\boldsymbol{r}, R)$ in Fig. 5. In this way we can directly assess, in terms of observed quantities, in which over-density ranges the VVDS environment catalogue is reliable. We see that, for $R<5 h^{-1} \mathrm{Mpc}$, the observed over-density field underestimates the underlying parent density contrast in under-dense regions and overestimates it in over-dense ones. Only for $R \geq 5 h^{-1} \mathrm{Mpc}$ the observed over-densities trace the underlying true distribution in a fair way.

In short, the results of simulated VVDS observations presented in this section show that, on scales $R \geq 5 h^{-1} \mathrm{Mpc}$, 3D over-densities are essentially free from selection systematics at least for what concerns the unbiased identification of galaxy environments in both low and high density regions. Obviously, the representativeness of the measured environments with respect to the "universal" one is a different question. Since the volume probed is still restricted to one survey field, the dynamical range of the recovered over-densities may be affected by cosmic variance (e.g., Ilbert et al. 2006a). From now on we will use $3 \mathrm{D}$ over-densities as measured with a filter of size $R=5 h^{-1} \mathrm{Mpc}$.

\section{Results}

In this section we present our results on the dependence of galaxy colours from local density, luminosity and redshift. As Blanton et al. (2005) among other authors have shown, colour is the property that best correlates, together with luminosity, with local environment. For this analysis we use rest-frame $\left(u^{*}-g^{\prime}\right)$ colours, uncorrected for dust absorption, derived from rest-frame AB absolute magnitudes as computed in the $u^{*}$ and $g^{\prime}$ CFHTLSMEGACAM photometric system. The strategy adopted to derive rest-frame absolute magnitudes is described in detail in Ilbert et al. (2005), and we refer the reader to that paper for details. The CFHTLS-MEGACAM photometric system has been designed to match the SDSS filters as closely as possible, with the only exception of the $u^{*}$ filter, that is slightly wider than the SDSS $u^{\prime}$ filter (see Ilbert et al. 2006a). 


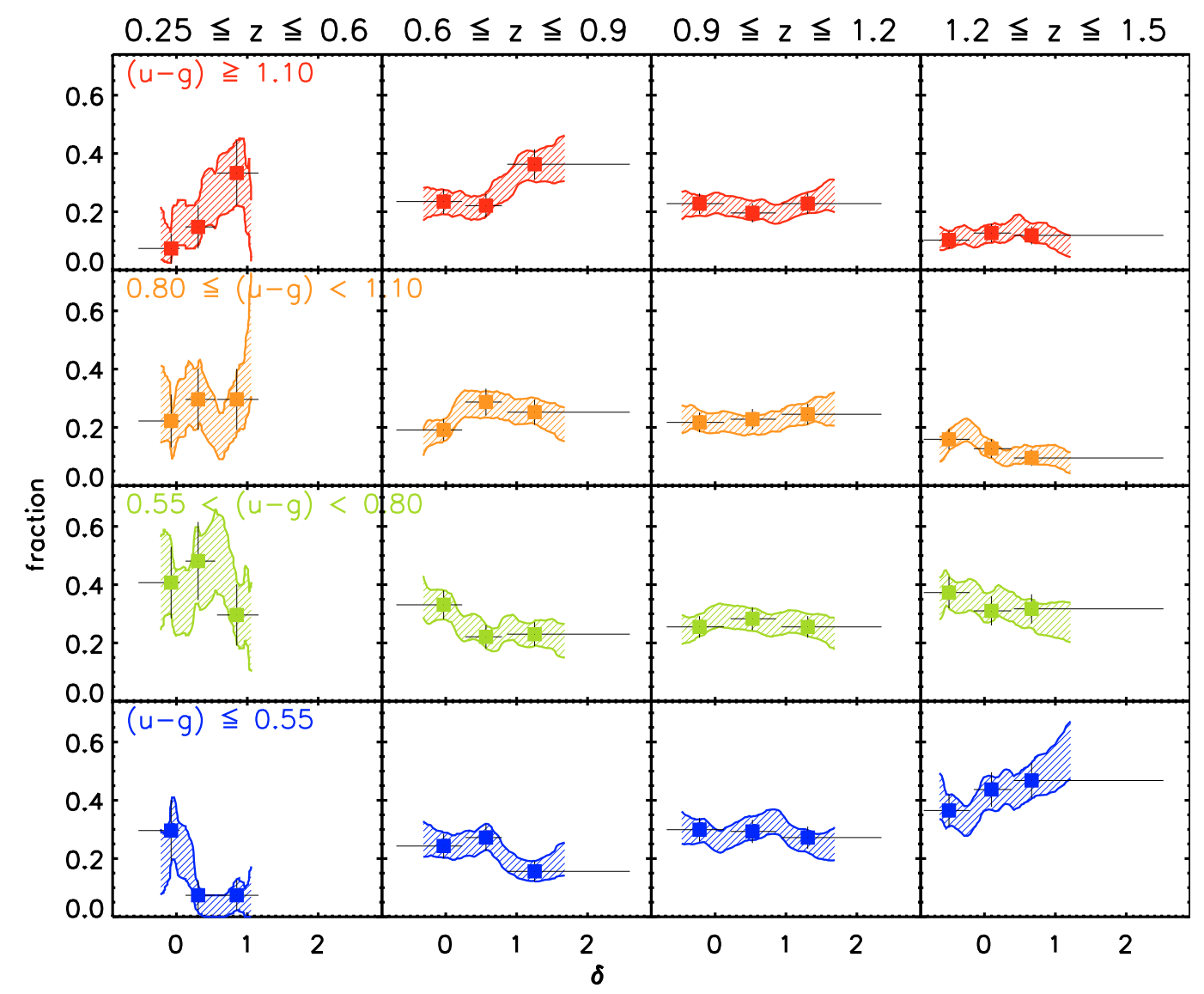

Fig. 6. The fraction of galaxies with luminosities greater than $\left(M_{B}-5 \log h\right)<-20$ and with rest-frame colours $\left(u^{*}-g^{\prime}\right) \geq 1.1,0.80 \leq\left(u^{*}-g^{\prime}\right)<1.1$, $0.55<\left(u^{*}-g^{\prime}\right)<0.8$ and $\left(u^{*}-g^{\prime}\right) \leq 0.55$ (from top to bottom) is plotted as a function of environment $\left(\delta\right.$ on $\left.R=5 h^{-1} \mathrm{Mpc}\right)$ in four different redshift intervals (from left to right, as indicated on the top). The horizontal bars indicate the amplitude of the bins in $\delta$ (i.e. the range spanned by the lower $5 \%$ and upper $95 \%$ percentile of the objects contained in each bin) while vertical error-bars represent a Poissonian $1 \sigma$ uncertainty. The shaded areas are obtained by smoothing the red (blue) fraction with an adaptive sliding box containing the same number of objects in each bin as the points marked explicitly.

We choose the $\left(u^{*}-g^{\prime}\right)$ colour because it brackets the Balmer break and is therefore particularly sensitive to galaxy properties (e.g., age, recent star formation and metallicity variations of the stellar population). Another advantage of this colour choice is that, given the range of filters available to our survey, rest-frame absolute magnitudes are better reconstructed (out to $z \sim 1.5$ ) in the $u^{*}$ and $g^{\prime}$ bands than in other redder filters (Ilbert et al. 2005).

\subsection{The colour-density relation: redshift and luminosity dependence}

We empirically defined 4 different galaxy "colour-types" on the basis of the following rest-frame colour criteria: $\left(u^{*}-g^{\prime}\right) \geq 1.1$, $0.80 \leq\left(u^{*}-g^{\prime}\right)<1.1,0.55<\left(u^{*}-g^{\prime}\right)<0.8$ and $\left(u^{*}-g^{\prime}\right) \leq 0.55$. Note that our reddest and bluest colour classes roughly correspond to the two colour peaks visible in the bimodal colour distribution shown in Fig. 10.

In Fig. 6 we plot the fraction of galaxies of each colourtype and with luminosities greater than $\left(M_{B}-5 \log h\right)<-20$ as a function of the environment (i.e. the density contrast $\delta$ ). The colour-density relation is portrayed at four different cosmic epochs. This figure shows one of the key observational results of our investigation: the significant redshift dependence of the colour-density relationship. In the lowest redshift bin $(0.25 \leq z \leq 0.60)$, the bluest (reddest) galaxies are preferentially located in low (high) density regions, the trend changing smoothly through intermediate colours. This trend, which is reminiscent of the well-known local morphology-density relationship, progressively disappears and possibly reverses in the highest redshift bin $(1.20 \leq z \leq 1.50)$.

On top of this trend, another important key feature is evident: the strong evolution in the mean fraction of the bluest galaxies as a function of cosmic time. The relative abundance of these objects increases with increasing redshift, in agreement with what found by other redshift surveys of the deep universe (e.g., Lilly et al. 1995; Lin et al. 1999). However Fig. 6 adds an important element to the picture: the fraction of blue galaxies increases with increasing redshift not only in rich environments but also in under-dense regions (see last row of Fig. 6).

We also explored the combined dependence of the colour-density relation on redshift and luminosity. To this purpose, we selected different samples of galaxies, using as luminosity thresholds the values $\left(M_{B}-5 \log h\right) \leq$ $-19.0,-19.5,-20.0,-20.5,-21.0$ respectively. For each of these samples the fractions of the reddest $\left(\left(u^{*}-g^{\prime}\right) \geq 1.10\right)$ and bluest $\left(\left(u^{*}-g^{\prime}\right) \leq 0.55\right)$ galaxies are shown in Fig. 7 as a function of $\delta$ in four different redshift bins.

Note that a) the survey flux limit $(I=24)$ forces us to restrict the analysis of the fainter samples only to the lower redshift bins, b) the limited area of the survey prevents us to collect adequate signal from bright objects in the lowest redshift bin, because the 


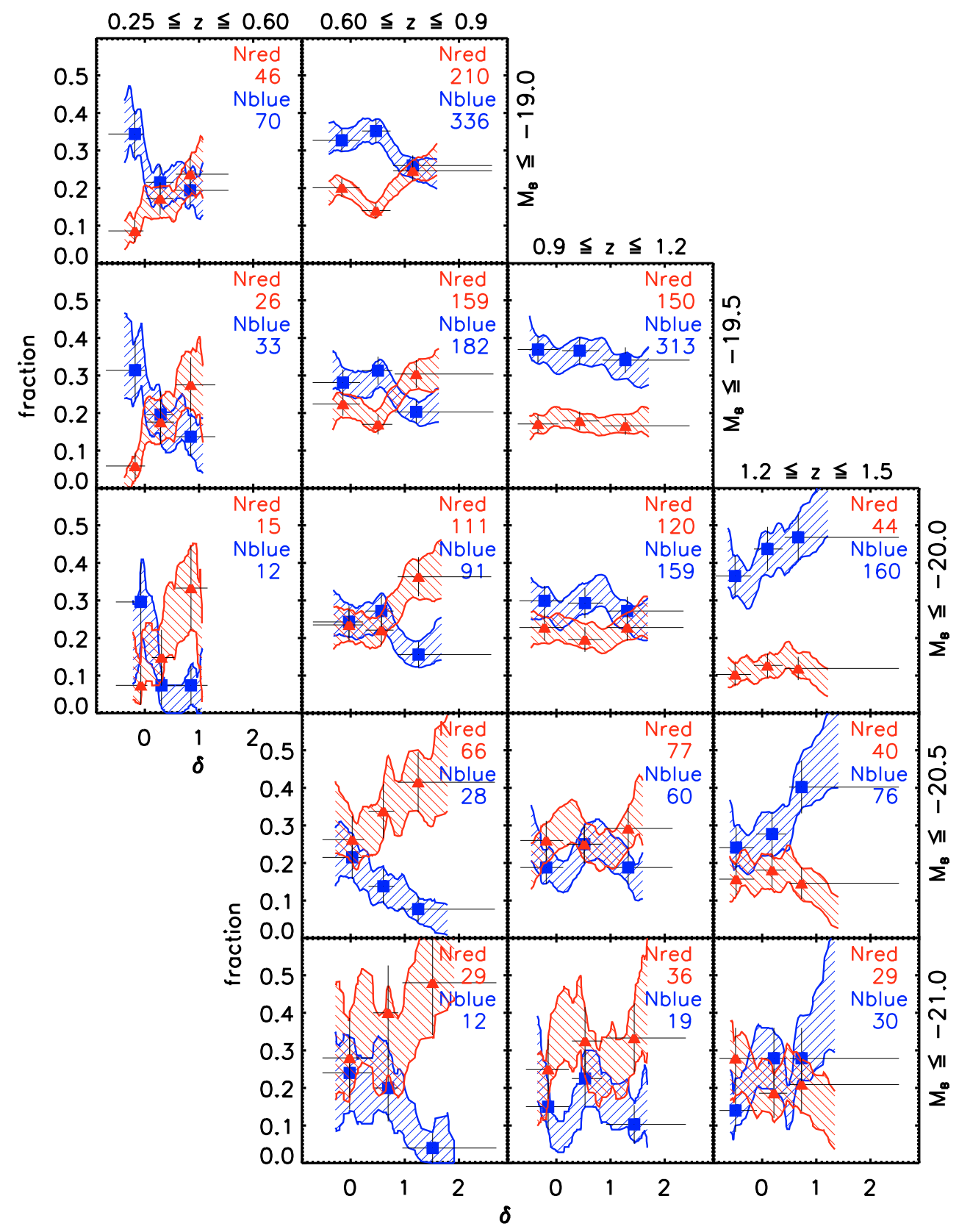

Fig. 7. The fraction of the reddest $\left(\left(u^{*}-g^{\prime}\right) \geq 1.10\right.$, triangles $)$ and bluest $\left(\left(u^{*}-g^{\prime}\right) \leq 0.55\right.$, squares $)$ galaxies is plotted as a function of the density contrast $\delta$ in different redshift intervals (columns, as indicated on top) and for different absolute luminosity thresholds (rows, as indicated on the right). The horizontal bars indicate the amplitude of the bins in $\delta$ (i.e. the range spanned by the lower 5\% and upper $95 \%$ percentile of the objects contained in each bin, the marked points being located at the median value) while vertical error-bars represent a Poissonian $1 \sigma$ uncertainty. The shaded areas are obtained by smoothing the reddest(bluest) fraction with an adaptive sliding box containing the same number of objects in each bin as the points marked explicitly. The number of red and blue galaxies in each redshift and luminosity bin is explicitly indicated in the corresponding panel.

volume explored at low redshift is not large enough to sample the exponentially decreasing bright end of the luminosity function.

While all the samples used in the first three redshift bins of Fig. 7 are indeed samples purely limited in absolute magnitude, the samples in the last redshift bin could be partially affected by colour incompleteness. Our survey is flux-limited at $I \leq 24$ and the $I$-band corresponds to $B$-band rest frame wavelength at redshift $z \sim 0.8$. For redshifts greater than this value the absolute magnitude cut-off that selects our samples will be a function of the colour of the galaxy population considered - see e.g., Fig. 2 in Ilbert et al. (2004). In particular while at $z \leq 1.2$ we can safely assume to be complete down to $\left(M_{B}-5 \log h\right) \sim-20.0$ irrespective of galaxy colours, when moving to the last redshift bin $1.2 \leq z \leq 1.5$ only the sample limited at $\left(M_{B}-5 \log h\right) \sim$ -21.0 is complete for galaxies whose colour is as red as that of evolved ellipticals observed in the local universe.

Therefore, the overall normalization of the $\left(M_{B}-5 \log h\right) \leq$ -20.0 and $\leq-20.5$ reddest samples in the last redshift bin should be considered as a lower limit.

As an additional test on the density estimates obtained with Eq. (2), we computed local densities also using a volume limited sample $\left(\left(M_{B}-5 \log h\right) \leq-20.0\right)$.

By using as the population to define the density contrast $\delta$ only galaxies brighter than a fixed $B$-band absolute magnitude, 


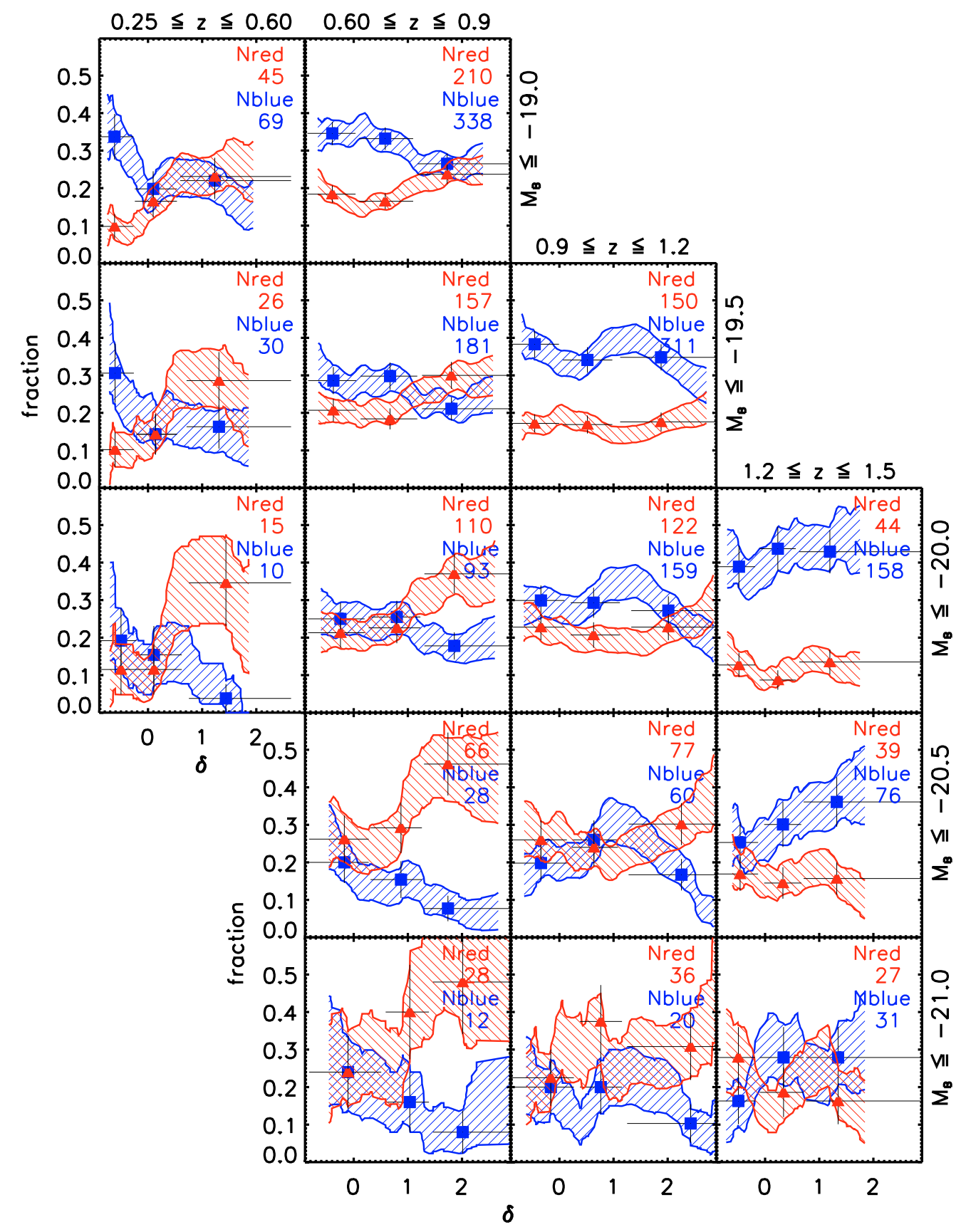

Fig. 8. As in Fig. 7, but computing local densities using only galaxies brighter than $\left(M_{B}-5 \log h\right)=-20.0$. See text for details.

we can drop the redshift dependent selection function $S\left(r, \mathcal{M}^{c}\right)$ in Eq. (2). This way the noise of the density estimate does not depend on redshift nor on the luminosity function, whose estrapolation at high redshifts is plagued by uncertainties. Futhermore we avoid introducing, especially at high redshifts, an artificial homogeneity in the galaxy distribution, as progressively brighter galaxies are used to trace the space distribution of the full galaxy population. The results obtained with this recipe are displayed in Fig. 8, that shows the same trends as in Fig. 7, although a bit noisier, especially in the first redshift bin where the density reconstruction is based on a much smaller number of galaxies than in Fig. 7.

Both Figs. 7 and 8 show that not only the colour segregation weakens as a function of redshift for galaxies of similar luminosity, but, at a fixed redshift, it strongly depends on luminosity: for progressively brighter galaxies the colour-density relationship, as we know it in the local universe, appears at earlier cosmic times.

To quantify the statistical significance of our findings, we fitted the points plotted in these figures with a linear relation ( $f=a+b \delta$, where $f$ is the fraction of red or blue galaxies). The $1 \sigma$ error bars obtained by fitting our data well agree with those obtained with randomization techniques. In this case we randomized 1000 times, for each redshift and luminosity bin, the distribution of $\delta$ among our galaxies, and for the randomized sample we plotted the quantities shown in Figs. 7 and 8, testing how often the linear fit to the randomized data would provide a slope as steep or steeper than the one measured from our data.

Figure 9 shows the slopes $b$ and the associated $1 \sigma$ error bars as a function of redshift, for red $\left(\left(u^{*}-g^{\prime}\right) \geq 1.1\right.$, triangles $)$ and blue $\left(\left(u^{*}-g^{\prime}\right) \leq 0.55\right.$, squares $)$ galaxies, for the three subsamples limited at $\left(M_{B}-5 \log h\right)=-19.0,-20.0,-21.0$ going from 


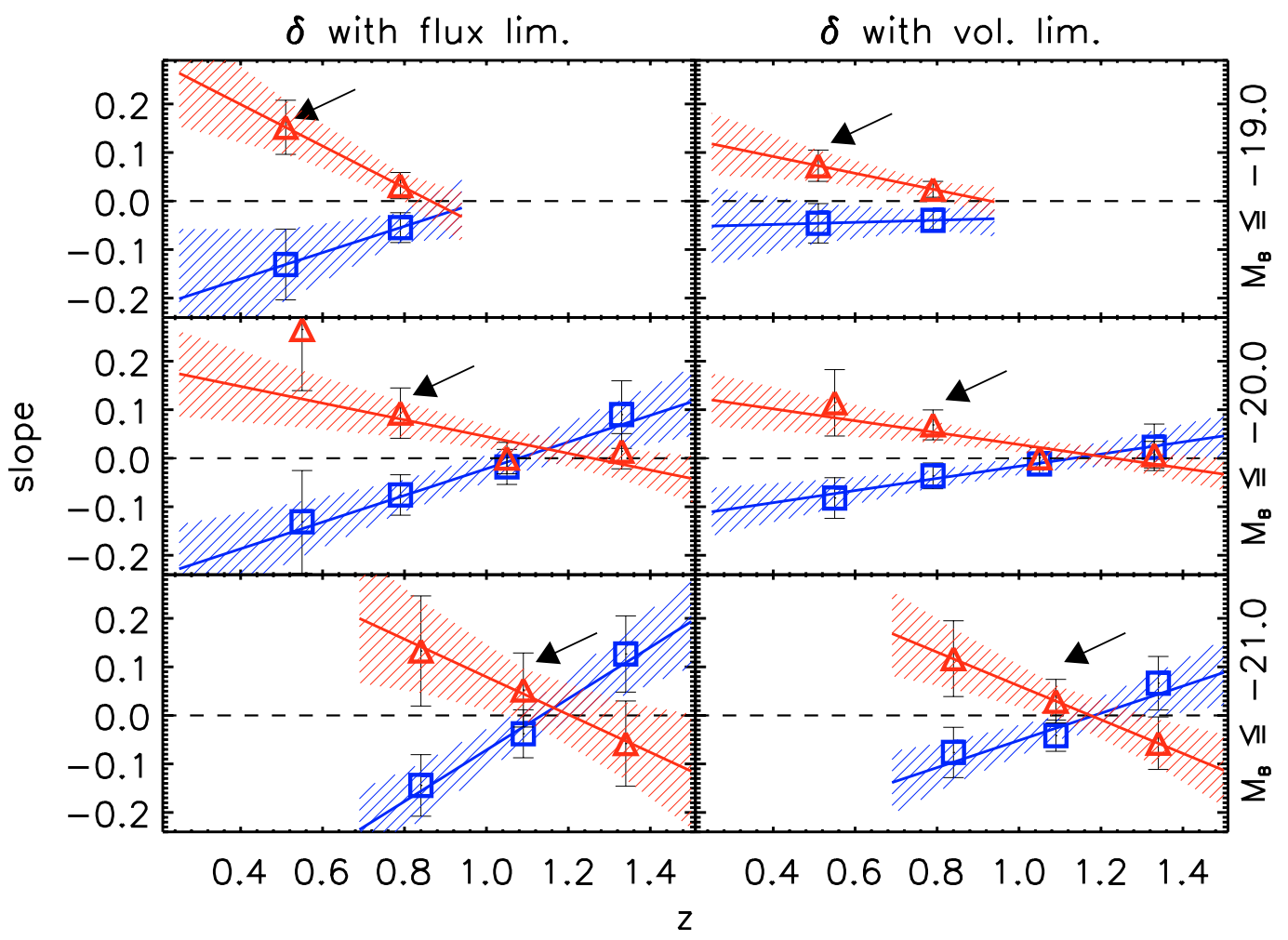

Fig. 9. Best fit slopes (and their associated $1 \sigma$ error bars) of the fraction of reddest $\left(\left(u^{*}-g^{\prime}\right) \geq 1.10\right.$, triangles) and bluest $\left(\left(u^{*}-g^{\prime}\right) \leq 0.55\right.$, squares) galaxies as a function of $\delta$ for the four different redshift bins of Fig. 7 (left column) and of Fig. 8 (right column). From top to bottom different limits in absolute magnitude are considered, as indicated on the vertical label on the right. In each panel the straight lines (and the shaded error bar area associated) are the result of the linear fit to the points shown. The black arrows indicate the redshift bin where the colour-density relationship, as we know it in the local universe, appears for the first time.

top to bottom. Left panel refers to Fig. 7, i.e. when density contrast is estimated using the full flux limited sample, while right panel refers to Fig. 8. The black arrows indicate the redshift bin where the colour-density relationship, as we know it in the local universe, appears for the first time.

Considering the results obtained using a flux limited sample to estimate densities, at low redshift $(0.25 \leq z \leq 0.60)$ we find that for all the subsamples considered red galaxies are preferentially located in high density regions (positive slope), while blue galaxies are preferentially located in low density regions (negative slope). These slopes are different from zero at more than $2 \sigma$ level for red galaxies and nearly $2 \sigma$ level for blue ones, and their relative difference for each panel in the first column of Fig. 7, is significant at the $2.5-3 \sigma$ level. In the next redshift bin $(0.60 \leq z \leq 0.90)$, significant differences (at $2.5 \sigma$ level) between the slopes of the fraction of red and blue galaxies as a function of $\delta$ are present only for galaxies brighter than $\left(M_{B}-5 \log h\right)=$ -20.0 . Viceversa for galaxies fainter than $\left(M_{B}-5 \log h\right)=-20.0$ no significant trend with density is seen for the fractions of both red and blue galaxies. At even higher redshift $(0.90 \leq z \leq 1.20)$, some difference between the slopes of the fraction of red and blue galaxies as a function of $\delta$ is visible only for the very brightest galaxies $\left(\left(M_{B}-5 \log h\right) \leq-21.0\right)$, although at a low level of significance $(1 \sigma)$, also because of the small statistics. Finally in the highest redshift bin explored $(1.20 \leq z \leq 1.50)$, for the very brightest galaxies $\left(\left(M_{B}-5 \log h\right) \leq-21.0\right)$, the slope of the fraction of red(blue) galaxies as a function of $\delta$ is even negative(positive), though at $1(1.6) \sigma$ level.

When the density contrast is estimated using the $\left(M_{B}-\right.$ $5 \log h) \leq-20.0$ sample the significance of our findings is slightly lower. This is partially due to the noisier estimates of densities, at least in the first redshift bins, but the substance of our results does not change, as one can estimate from Figs. 8 and 9.

\subsection{The colour-magnitude diagram: redshift and density dependence}

In this section we explore the evolution of the distribution of galaxies in the colour-magnitude plane $\left(u^{*}-g^{\prime}\right)$ vs. $\left(M_{B}-5 \log h\right)$ as a function of both redshift and environment, further expanding the correlations discussed in the previous section.

In Fig. 10 the first 3 columns show the isodensity contours of the distribution of galaxies in different redshift ranges (from top to bottom as indicated on the right) and for different environments (from left to right as indicated on top). The difference between the over-dense and under-dense colour-magnitude distributions is shown in the fourth column.

The first column shows that the bimodal distribution of galaxies in colour space, well established in the local universe (e.g., Strateva et al. 2001), persists out to the highest redshift investigated $(z \sim 1.5)$. This analysis confirms and extends at higher redshifts previous results obtained with photometric redshifts out to $z=1$ (e.g., Bell et al. 2004b; Nuijten et al. 2005). A more detailed analysis of the bimodality of our sample is presented in Franzetti et al. (2006).

The second and third columns of Fig. 10 show that bimodality survives irrespective of environment out to $z \sim 1.5$. Beyond the bimodality, it is important to notice that the location of the colour "gap" between the red and blue peaks appears to be roughly constant and insensitive to environment at all redshifts. This result justifies a posteriori our choice of a fixed colour 


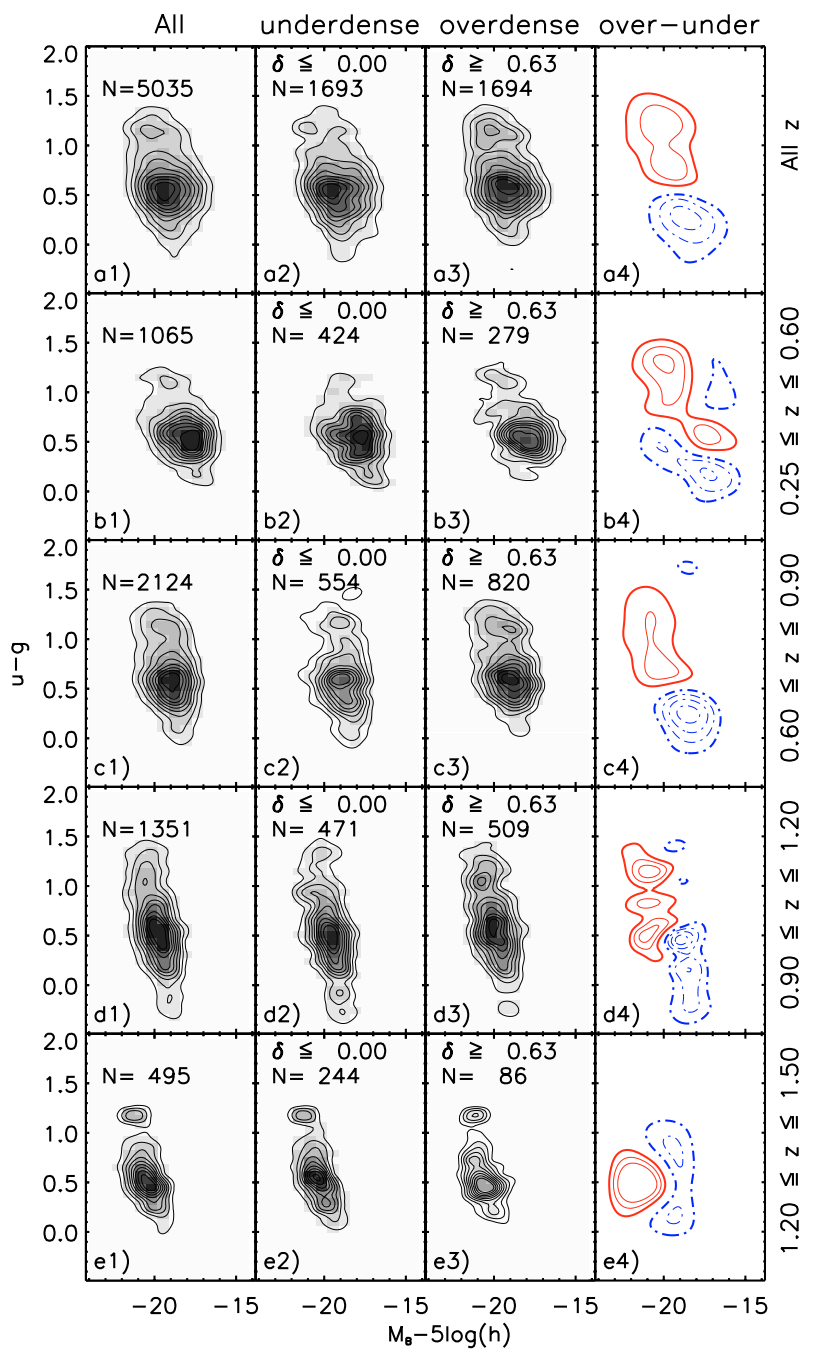

Fig. 10. The first 3 columns show the isodensity contours of the distribution of galaxies in the $\left(u^{*}-g^{\prime}\right)$ vs. $\left(M_{B}-5 \log h\right)$ plane for different redshift ranges (from top to bottom as indicated on the right) and for different environments (from left to right as indicated on top). The grey scale is normalized, for each panel, to the total number of objects contained in that panel. The difference between the over-dense and under-dense colour-magnitude distributions is shown in the 4 th column. $1-2-3-\sigma$ levels of significance in the difference are shown using red continuous lines (positive differences), and blue dotted lines (negative differences). The thicker lines correspond to $1 \sigma$ level.

threshold in splitting the total sample into a blue and a red subset.

We can discriminate finer environmental dependencies imprinted in the bimodal colour distribution by plotting the difference between the over- and under-dense colour-magnitude distributions. The fourth column of Fig. 10 shows that the colour-magnitude distribution is not universal but strongly depends upon environment. At low redshift, and for any luminosity, there is a prominent excess of red objects in over-dense regions, while under-dense regions are mostly populated by blue galaxies. On the other hand, and most interestingly, moving towards higher redshifts the relative ratio of the two peaks of the bimodal distribution becomes mostly insensitive to environment (at $0.9<z<1.2$ ) with the hint of the development of a more pronounced peak of blue galaxies in high density regions in the last redshift bin $(1.2<z<1.5)$. Stated differently, the bulk of the red population in the interval $0.9<z<1.2$ is found to be equally distributed in different environments, with the brightest red sample, however, still biased toward high density regions. At higher redshifts, $1.2<z<1.5$, even the brightest red galaxies are not preferentially found in over-dense regions, which, instead, become mostly populated by bright blue objects.

\section{Discussion}

The most striking result of this study is displayed in Fig. 7: the colour-density relation evolves dramatically as a function of cosmic time. While at the remotest epochs explored $(z \sim 1.5)$ even the most luminous red galaxies do not reside preferentially in high density environments, as cosmic time goes by, the environmental dependence of galaxy colours progressively builds up, earlier for brighter galaxies and later for fainter galaxies. For example, the faintest red galaxies $\left(M_{B}<-19.0+5 \log h\right)$ are preferentially located in high density environments only in the nearest redshift bin investigated, confirming earlier hints in this direction obtained with photometric redshifts (e.g., Kodama et al. 2004; Yee et al. 2005). Vice versa in the redshift bin $0.9 \leq z \leq 1.2$ all but the most luminous red galaxies, $\left(M_{B}-5 \log h\right)<-20.5$, show a flat colour-density relation. In the highest redshift bin even the brightest red objects are not preferentially found in rich environments as suggested by the fact that the slope of the colourdensity relation for red objects turns negative ( $1 \sigma$ effect). In this redshift bin there is a suggestion that the percentage of bluest galaxies increases in the highest density contrast regions, hinting that in remote look-back times the star formation activity was higher in high density peaks than in low density regions, a property reminiscent of a similar characteristic of Ly-break galaxies (Foucaud et al. 2003). We conclude that at $z \sim 1$ there is evidence of absence of the colour-density relation for medium luminosity galaxies. Moreover, there are hints that the well established local trend, which progressively disappears even for the brightest galaxies in our sample after $z \sim 1.2$, eventually reverses in the highest redshift bins investigated $(\sim 1 \sigma$ effect).

Not only the slope of the colour-density has changed, but also the overall normalization. The decrease of the relative fraction of the bluest galaxies from high redshift to the present day is well established in literature and can be traced back to the observations of the increase in the abundance of star-forming galaxies in high redshift clusters (see Butcher \& Oemler 1984). In our study, we find that this trend holds true also in low density environments.

Figures 7 and 8 show that the fraction of the bluest galaxies brighter than $\left(M_{B}-5 \log h\right)<-20$ which inhabit low density regions at $1.2 \leq z \leq 1.5$ decreases on the mean as cosmic time goes by. On the other hand, over the same redshift range, the relative abundance of blue objects has changed by nearly one order of magnitude in over-dense environments. Similarly the reddest galaxies experience a faster increase with cosmic-time in overdense regions than in under-dense regions. This result indicates that the mechanisms governing galaxy formation and evolution operate with different timescales in different environments.

We can interpret these findings by making some simplifying hypothesis. Let's assume, to first order, that the adopted colour classes are a proxy for different star formation histories, bluer galaxies having experienced relatively recent star formation. In this case, the observed strong time dependence of the colourdensity relation implies that star formation is differentially suppressed in high and low density regions. For galaxies of similar luminosity the drop in star formation rate occurred earlier in higher density environments, resulting in the red excess observed at present epoch, and progressively later in lower density 
environments, i.e. in the field, where a larger blue component is still observed. This result suggests that some environment driven mechanism may be at work. The drop in star formation is also a function of luminosity (and therefore probably mass): truncation mechanisms are more efficient in brighter systems than in fainter ones.

By further assuming that it is empirically possible to define early and late type galaxies in an unbiased, model-independent way by exploiting the bimodality of the galaxy colour distribution out to the highest redshift investigated (e.g., Bell et al. 2004b) our findings would imply a change in the morphologydensity relation (Dressler 1980) for galaxies brighter than $M_{B}^{*}(z=0)(\sim-20+5 \log h$, see Ilbert et al. 2005) at $z \sim 1$ and for brighter ones at $z \sim 1.5$.

Even if there are evidences that most colour selected red galaxies are dominated by an old stellar population from $z=0$ (e.g., Strateva et al. 2001) up to $z \sim 1$ (Bell et al. 2004a), the situation at larger redshift is unclear and a substantial fraction of red galaxies could be dusty starbursts (e.g., Cimatti et al. 2003). Therefore, we caution that our results should be interpreted as an upper limit on the distribution of red passive objects. If red dusty starburst galaxies inhabit high density regions, then the deficit of red old objects in high density environments at high redshift should be even stronger than that estimated in our analysis.

From an observational side our analysis well agrees with the so called downsizing scenario, first suggested by Gavazzi et al. (1996) and Cowie et al. (1996), but modified to take into account the observed environmental dependence. According to our observations, star formation activity is not only progressively shifted to smaller systems, but also from higher to lower density environments.

This result agrees remarkably well with our findings (obtained with the same sample) about the significant evolution of galaxy biasing out to $z \sim 1.5$ (Marinoni et al. 2005). In that study we showed that we live in a special epoch in which the distribution of galaxies with $M_{B}-5 \log h \sim-20$ traces the underlying mass distribution on scales $R \geq 5 h^{-1} \mathrm{Mpc}$ while, in the past, the two fields were progressively dissimilar and the relative biasing higher. In other words, while at high redshift bright galaxies formed preferentially in the high matter-density peaks, as the Universe ages, galaxy formation begins to take place also in lower density environments. This result on biasing evolution provides a simple and intuitive way to introduce environment in the original downsizing picture: brighter galaxies start forming stars earlier and preferentially in higher density environments.

From a theoretical perspective, in models of hierarchical galaxy formation (Kauffmann et al. 1993; Somerville \& Primack 1999; Cole et al. 2000), it is assumed that massive galaxies, which accreted earlier and in a biased way with respect to the underlying matter density field, have their hot gas reservoir depleted, which results in a premature truncation of the star formation activity relative to field galaxies. Our findings about galaxy biased formation coupled with simple assumptions for the faster gradual decline of the star formation activity of galaxies in dense environments are able to explain, in a qualitative way, the observed evolution of the colour-density relation, i.e. the faster progressive building up of bright red galaxies in high density environments and the slower evolution for the fainter galaxy population.

Several physical processes have been proposed that may account for a consumption/expulsion/evaporation of gas in high density environments: ram pressure stripping (Gunn \& Gott 1972), galaxy-galaxy merging (Toomre \& Toomre 1972), strangulation (Larson et al. 1980) and harassment (Moore et al. 1996).
However the scale over which the over-density field of the deep universe is reconstructed in this paper, prevents us from concluding on the possible local causes of the observed evolution in the colour-density relation. In other words, we cannot discriminate if the mechanism responsible for the differential evolution is acting also at large distance from the high density cluster core regions (e.g., Balogh et al. 1997).

The strengthening of the colour-density relation as a function of cosmic time implies that the colour distribution has been tightly coupled to the underlying density field at least over the past 9 Gyr. The effects of this coupling are evident in Fig. 10 where we show the different evolution of the colour-magnitude distribution in high and low density environments. The early epoch flatness of the colour-density relation causes the bimodal colour distribution in high density regions to mirror the one in poor environments. However, as time goes by, the colour-density relation strengthens and the bimodal distribution gradually develops the present-day asymmetry between a red peak more prominent in high density environments and a blue one mostly contributed by field galaxies.

Besides evolution in the gradient and amplitude of the colour-magnitude distribution, one may however notice a few interesting features which are stable across different cosmic epochs. The peak position of the red population remains nearly unchanged in both over- and under-dense environments out to $z \sim 1.5$. This implies that a population of red objects of bright luminosities and in different environments is already well evolved by redshift 1.5 (see also Le Fèvre et al. 2006, in preparation). This finding may be easily, and perhaps most naturally, interpreted as supporting evidence for a scenario in which old, massive, quiescent objects were already in place at redshift 1.5 and have undergone very little evolution since then. Figure 10 shows another interesting similarity between the low and high redshift universe: brighter galaxies are redder both in low and high density environments and this holds true at all redshifts investigated. We thus find that the colour-magnitude relation, which has been well investigated in clusters up to $z \sim 1$ (e.g., Visvanathan $\&$ Sandage 1977; Bower et al. 1992; Holden et al. 2004; Tanaka et al. 2005) also applies, irrespective of cosmic epochs, to galaxies populating very under-dense environments.

\section{Conclusions}

The size (6582 galaxies with good quality redshifts), depth $\left(I_{A B} \leq 24\right)$ and redshift sampling rate $(20 \%$ on the mean) of the VVDS-02h deep survey allowed us to reconstruct the 3D galaxy environment on relatively local scales $\left(R=5 h^{-1} \mathrm{Mpc}\right)$ up to redshift $z=1.5$ and to study the colour distribution as a function of density, luminosity and look-back time.

Environmental studies at high redshift have traditionally focused only on high density regions (galaxy clusters), and/or have been based on galaxy position inferred using photometric redshifts. Our study represents the first attempt to use a purely flux-limited redshift survey to explore the primordial appearance of the colour-density and colour-magnitude diagrams from the densest peaks of the galaxy distribution down to very poor environments and faint magnitudes.

We have paid particular attention to calibrate our density reconstruction scheme, and the extensive simulations presented in this paper enable us to determine the redshift ranges and smoothing length scales $R$ over which our environmental estimator is not affected by the specific VVDS observational constraints. These include intrinsic limitations in recovering real space positions of galaxies (peculiar velocities contaminations, 
spectroscopic accuracy...), survey geometrical constraints, sampling and instrumental selections effects. We conclude that we reliably reproduced the underlying real galaxy environment on scales $R \geq 5 h^{-1}$ Mpc out to $z=1.5$.

Our findings can be summarised as follows:

a) The colour-density relation shows a dramatic change as a function of cosmic time. While at the lowest redshifts explored we confirm the existence of a strong colour-density relation, with the fraction of the red(/blue) galaxies increasing(/decreasing) as a function of density, at previous epochs blue and red galaxies seem to be mostly insensitive to the surrounding environment, with a nearly flat distribution of the fraction of the bluest and reddest objects over the whole over-density range. The absence of the colour-density relation at the highest redshift bins investigated implies that quenching of star formation was more efficient in high density regions.

b) The evolution of the colour-density relation depends on luminosity. Not only, at fixed luminosity, there is a progressive decrease of red objects as a function of redshift in high density regions, but also, at fixed redshift, there is a progressive decrease of fainter red galaxies. This result implies that star formation ends at earlier cosmic epochs for more luminous/massive galaxies.

c) The relative fraction of the bluest objects was higher in the past in both high and low density environments and for both more luminous and fainter galaxies. However, the observed drop in the star formation rate of blue objects in poor environments is weaker than in high density environments and is weaker for fainter galaxies. This result indicates that star formation rate continues to be substantial at the present day in field galaxies, and especially in the fainter ones.

d) The bimodal $\left(u^{*}-g^{\prime}\right)$ colour-magnitude distribution shows a marked dependence on environment. While locally the colour-magnitude diagrams in low and high density regions are significantly different, in the highest redshift bin investigated the two distributions mirror each other. This suggests that we have sampled the relevant time-scales over which physical nurture processes have conspired to shape up the present-day density dependence of the colour-magnitude distribution.

We conclude that the colour-density and colour-magnitudedensity relations are not the result of initial conditions imprinted early on during the primordial stages of structure formation and then frozen during subsequent evolution. Our results suggest a scenario whereby both time evolving genetic information (galaxy biased formation) and complex environmental interaction (star formation quenching) concurred to build up these relations.

We remark that the scale $\left(5 h^{-1} \mathrm{Mpc}\right)$ over which the overdensity field of the deep universe is reconstructed in this paper prevents a straightforward extrapolation of our results down to cluster scales.

In a companion paper (Ilbert et al. 2006b) we focus on the analysis of the environmental effects on the galaxy Luminosity Function at high redshift. Complementary analysis based on data from various ongoing deep redshift survey, such as the zCOSMOS (Lilly et al. 2006) and DEEP2 surveys (Davis et al. 2003), may help in sheding further light on our findings. Indeed, disentangling the role of environmental conditions on the evolution of galaxy structural parameters and especially finding how to accommodate present observations within the context of the competing models of galaxy formation and evolution will present a fascinating challenge for both observers and theorists over the next few years.

Acknowledgements. We thank Darren Croton, Frank van den Bosch, Stefano Andreon and Simone Weinmann for stimulating discussions. We thank also the referee for helpful comments which improved the content of the paper. This research has been developed within the framework of the VVDS consortium and it has been partially supported by the CNRS-INSU and its Programme National de Cosmologie (France), by the Italian Ministry (MIUR) grants COFIN2000 (MM02037133) and COFIN2003 (num.2003020150) and by PRIN-INAF 2005 (CRA 1.06.08.10). CM also acknowledges financial support from the Region PACA. The VLT-VIMOS observations have been carried out on guaranteed time (GTO) allocated by the European Southern Observatory (ESO) to the VIRMOS consortium, under a contractual agreement between the Centre National de la Recherche Scientifique of France, heading a consortium of French and Italian institutes, and ESO, to design, manufacture and test the VIMOS instrument.

\section{References}

Abbas, U., \& Sheth, R. K. 2005, MNRAS, 364, 1327

Balogh, M., Eke, V., Miller, C., et al. 2004a, MNRAS, 348, 1355

Balogh, M. L., Baldry, I. K., Nichol, R., et al. 2004b, ApJ, 615, L101

Balogh, M. L., Morris, S. L., Yee, H. K. C., Carlberg, R. G., \& Ellingson, E. 1997, ApJ, 488, L75

Bell, E. F., McIntosh, D. H., Barden, M., et al. 2004a, ApJ, 600, L11

Bell, E. F., Wolf, C., Meisenheimer, K., et al. 2004b, ApJ, 608, 752

Blaizot, J., Wadadekar, Y., Guiderdoni, B., et al. 2005, MNRAS, 360, 159

Blanton, M. R., Eisenstein, D., Hogg, D. W., Schlegel, D. J., \& Brinkmann, J. 2005, ApJ, 629, 143

Bottini, D., Garilli, B., Maccagni, D., et al. 2005, PASP, 117, 996

Bower, R. G., Lucey, J. R., \& Ellis, R. S. 1992, MNRAS, 254, 601

Butcher, H., \& Oemler, A. 1984, ApJ, 285, 426

Cimatti, A., Daddi, E., Cassata, P., et al. 2003, A\&A, 412, L1

Cole, S., Lacey, C. G., Baugh, C. M., \& Frenk, C. S. 2000, MNRAS, 319, 168

Cooper, M. C., Newman, J. A., Madgwick, D. S., et al. 2005, ApJ, 634, 833

Cowie, L. L., Songaila, A., Hu, E. M., \& Cohen, J. G. 1996, AJ, 112, 839

Davis, M., Faber, S. M., Newman, J., et al. 2003, in Discoveries and Research Prospects from 6- to 10-Meter-Class Telescopes II, ed. P. Guhathakurta, Proc. SPIE, 4834, 161

Dressler, A. 1980, ApJ, 236, 351

Foucaud, S., McCracken, H. J., Le Fèvre, O., et al. 2003, A\&A, 409, 835

Franzetti, et al. 2006, A\&A, submitted [arXiv: astro-ph/0607075]

Gavazzi, G., Pierini, D., \& Boselli, A. 1996, A\&A, 312, 397

Gómez, P. L., Nichol, R. C., Miller, C. J., et al. 2003, ApJ, 584, 210

Gunn, J. E., \& Gott, J. R. I. 1972, ApJ, 176, 1

Guzzo, L., Cassata, P., Finoguenov, A., \& the COSMOS collaboration 2006, ApJS, in press

Hatton, S., Devriendt, J. E. G., Ninin, S., et al. 2003, MNRAS, 343, 75

Hogg, D. W., Blanton, M. R., Brinchmann, J., et al. 2004, ApJ, 601, L29

Holden, B. P., Stanford, S. A., Eisenhardt, P., \& Dickinson, M. 2004, AJ, 127, 2484

Ilbert, O., Tresse, L., Arnouts, S., et al. 2004, MNRAS, 351, 541

Ilbert, O., Tresse, L., Zucca, E., et al. 2005, A\&A, 439, 863

Ilbert, et al. 2006a, A\&A, submitted [arXiv: astro-ph/0603217]

Ilbert, et al. 2006b, A\&A, submitted [arXiv: astro-ph/0602329]

Iovino, A., McCracken, H. J., Garilli, B., et al. 2005, A\&A, 442, 423

Kaiser, N. 1987, MNRAS, 227, 1

Kauffmann, G., White, S. D. M., \& Guiderdoni, B. 1993, MNRAS, 264, 201

Kodama, T., Yamada, T., Akiyama, M., et al. 2004, MNRAS, 350, 1005

Larson, R. B., Tinsley, B. M., \& Caldwell, C. N. 1980, ApJ, 237, 692

Le Fèvre, O., Saisse, M., Mancini, D., et al. 2003, in Instrument Design and Performance for Optical/Infrared Ground-based Telescopes, ed. M. Iye, \& A. F. M. Moorwood, Proc. SPIE, 4841, 1670

Le Fèvre, O., Mellier, Y., McCracken, H. J., et al. 2004a, A\&A, 417, 839

Le Fèvre, O., Vettolani, G., Paltani, S., et al. 2004b, A\&A, 428, 1043

Le Fèvre, O., Vettolani, G., Garilli, B., et al. 2005, A\&A, 439, 845

Le Fèvre, et al. 2006, in preparation

Lilly, S. J., Tresse, L., Hammer, F., Crampton, D., \& Le Fevre, O. 1995, ApJ, 455,108

Lilly, et al. 2006, in prep.

Lin, H., Yee, H. K. C., Carlberg, R. G., et al. 1999, ApJ, 518, 533

Marinoni, C., Monaco, P., Giuricin, G., \& Costantini, B. 1999, ApJ, 521, 50

Marinoni, C., Hudson, M. J., \& Giuricin, G. 2002, ApJ, 569, 91

Marinoni, C., Le Fèvre, O., Meneux, B., et al. 2005, A\&A, 442, 801

McCracken, H. J., Radovich, M., Bertin, E., et al. 2003, A\&A, 410, 17

Mo, H. J., \& White, S. D. M. 1996, MNRAS, 282, 347 
Moore, B., Katz, N., Lake, G., Dressler, A., \& Oemler, A. 1996, Nature, 379, 613

Nuijten, M. J. H. M., Simard, L., Gwyn, S., \& Röttgering, H. J. A. 2005, ApJ, 626, L77

Oke, J. B., \& Gunn, J. E. 1983, ApJ, 266, 713

Poggianti, B. M., Smail, I., Dressler, A., et al. 1999, ApJ, 518, 576

Pollo, A., Meneux, B., Guzzo, L., et al. 2005, A\&A, 439, 887

Postman, M., Franx, M., Cross, N. J. G., et al. 2005, ApJ, 623, 721

Postman, M., \& Geller, M. J. 1984, ApJ, 281, 95

Smith, G. P., Treu, T., Ellis, R. S., Moran, S. M., \& Dressler, A. 2005, ApJ, 620, 78

Somerville, R. S., \& Primack, J. R. 1999, MNRAS, 310, 1087

Spitzer, L. J., \& Baade, W. 1951, ApJ, 113, 413

Strateva, I., Ivezić, Ž., Knapp, G. R., et al. 2001, AJ, 122, 1861

Tanaka, M., Kodama, T., Arimoto, N., et al. 2005, MNRAS, 362, 268

Toomre, A., \& Toomre, J. 1972, ApJ, 178, 623

Visvanathan, N., \& Sandage, A. 1977, ApJ, 216, 214

Weinmann, S. M., van den Bosch, F. C., Yang, X., \& Mo, H. J. 2006, MNRAS, 366, 2

Whitmore, B. C., Gilmore, D. M., \& Jones, C. 1993, ApJ, 407, 489

Yee, H. K. C., Hsieh, B. C., Lin, H., \& Gladders, M. D. 2005, ApJ, 629, L77 Zabludoff, A. I., \& Mulchaey, J. S. 1998, ApJ, 498, L5

1 INAF - Osservatorio Astronomico di Brera, via Brera 28, Milan, Italy; e-mail: olga.cucciati@brera.inaf.it

2 Universitá di Milano-Bicocca, Dipartimento di Fisica, Piazza della Scienza 3, 20126 Milano, Italy

3 Centre de Physique Théorique, UMR 6207 CNRS-Université de Provence, 13288 Marseille, France
4 Università di Bologna, Dipartimento di Astronomia, via Ranzani 1, 40127 Bologna, Italy

5 Laboratoire d'Astropysique de Marseille, UMR 6110 CNRSUniversité de Provence, BP 8, 13376 Marseille Cedex 12, France

${ }^{6}$ INAF - Osservatorio Astronomico di Bologna, via Ranzani 1, 40127 Bologna, Italy

7 IASF - INAF, via Bassini 15, 20133 Milano, Italy

8 Institut d'Astrophysique de Paris, UMR 7095, 98 bis Bvd Arago, 75014 Paris, France

9 Observatoire de Paris, LERMA, 61 Avenue de l'Observatoire, 75014 Paris, France

10 IRA - INAF, via Gobetti 101, 40129 Bologna, Italy

11 INAF - Osservatorio Astronomico di Roma, via di Frascati 33, 00040 Monte Porzio Catone, Italy

${ }^{12}$ Laboratoire d'Astrophysique de l'Observatoire Midi-Pyrénées (UMR 5572), 14 avenue E. Belin, 31400 Toulouse, France

13 INAF - Osservatorio Astronomico di Capodimonte, via Moiariello 16, 80131 Napoli, Italy

14 Max Planck Institut fur Astrophysik, 85741 Garching, Germany

15 European Southern Observatory, Karl-Schwarzschild-Strasse 2, 85748 Garching bei München, Germany

16 Integral Science Data Centre, ch. d’Écogia 16, 1290 Versoix, Switzerland

17 Geneva Observatory, ch. des Maillettes 51, 1290 Sauverny, Switzerland 